of the London Lock Hospital, which appeared in the Lancet for June 16,1917 , and which is reproduced in extenso in this number of our French contemporary. Darier has found McDonagh's treatment, in anything like his doses; so intensely painful that he and others have had to give it up. Nevertheless, the treatment is considered to be of such interest and importance to French readers that the Lancet article has been translated into their language.

ERNEST THOMSON.

(9) George, Edgar J., and Toren, Julius A. (Chicago).-An ocular therapeutic lamp. Ophthal. Record, May, 1917.

(9) George and Toren describe their leucodescent or therapeutic lamp in the treatment of corneal ulcers, iritis, episcleritis, and ciliary neuralgia.

The lamp consists of an aluminium parabolic reflector containing a 50-Watt electric light with a violet glass globe. The lamp is mounted on an adjustable stand with an adjustable face rest. The lamp and face must be adjusted so that the cornea is $60 \mathrm{~mm}$. from the front of the bulb (which is the focal distance of the reflector and will give a temperature of $170^{\circ} \mathrm{F}$. in 15 minutes), and must be accurately centered; $170^{\circ} \mathrm{F}$. was found to be the most effective temperature for the ocular diseases mentioned. This degree of heat. can only be tolerated by using the violet glass globe. The minimum thermal death-point for the staphylococcus is $149^{\circ} \mathrm{F}$. for 15 minutes; $158^{\circ} \mathrm{F}$. for 10 minutes; and $176^{\circ} \mathrm{F}$. for 5 minutes. For the streptococcus it is $129^{\circ} \mathrm{F}$. for 15 minutes and $113^{\circ} \mathrm{F}$. for 15 minutes for the pneumococcus.

The patient looks directly and constantly at the centre of the bulb with wide-open eye, but the lid is lowered at intervals to permit moistening of the eye. When the lamp is properly placed and adjusted the patients can take their own treatment. In cases of recent injury this form of treatment should be used as a prophylactic against infection.

J. Jameson Evans.

\title{
BOOK NOTICES
}

Malingering; or, the Simulation of Disease, by A. BASSETT JONES and J. LLEWELLYN LLEWELLYN; with a chapter on Malingering in relation to the Eye, by W. M. BEAUMONT. Pp. 708. Price 25s. London: William Heinemann. 1917.

Jones and Llewellyn have had considerable experience in the greatly increased legal work due to recent legislation of the type of 
the Workmen's Compensation Act, and are well qualified to deal with the subject of malingering. Now that conscription is in operation in this country, malingering has become very important from a military point of view. A very common form of malingering consists in the simulation of defective vision, and the authors have wisely arranged to have a special chapter written on this subject by an ophthalmic surgeon, W. M. Beaumont, of Bath, who has previously published a book on injuries of the eyes of the employed. This chapter occupies 91 pages and goes very fully into all the methods of detecting malingering and differentiating it from hysteria and real disease. Some of the tests may seem to the skilled observer superfluous, but it must be remembered that the evidence of malingering must be made clear and definite to a sufficient extent to convince others who have no special training and that nothing brings more discredit on the surgeon than an unwarranted diagnosis of malingering, to say nothing of the injustice that may follow to the unfortunate sufferer. In military work in connection with the classification of the soldier under the present British scheme, it will be found that a considerable number of men have found out that a visual acuity of less than 6/12 may be regarded as a passport to a safe job; thus the simulation chiefly found is an exaggeration of a natural defect, such as a moderate degree of myopia, and a refusal to admit any improvement up to that pitch even when correcting glasses are worn. It is not so easy to detect such cases, and in many the surgeon must be content to rely on his own observations as to the integrity of the fundus, the absence of symptoms of toxic amblyopia, etc. Beaumont rightly lays stress on the careful examination of the recruit in view to future claims for damage. He states: "The congenitally blind eye is one of the most prolific sources of ocular malingering. Even in the Army we often meet with binocular men with monocular sight, and in reply to our suggestion that the loss of function is not of recent inception, we are told that the eye must have been lost subsequently to enlistment, or they would not have been enrolled."

The simulation of night-blindness has given trouble of late. In a certain number of patients it is present as a congenital defect. The conditions of modern trench warfare are also responsible for fresh cases and increase in the congenital cases. A clever malingerer finds it an easy complaint to simulate, and one the simulation of which is by no means easy to detect. A test suggested by Beaumont is carried out by means of an EdridgeGreen lantern. The patient sits with his back to the lantern holding a sheet of white note paper in his hand, in such a way that the light falls upon it through the smallest perforation of the diaphragm of the lamp with an opaque glass in front of it. The perforation is increased in size until the paper is sufficiently illuminated for the 
patient to see it. The size of the perforation is noted, and then reversed examination is made, beginning with a full light, which is gradually reduced until the paper is no longer seen. Quantitative variations in the test may be made by removing the opaque glass. A note is made of the results, and the patient told to return on the following day, when a repetition of the test is made. The variations on the two days will be inappreciable in night-blindness, but extensive in malingering.

The whole article is full of suggestive hints on this most difficult subject and deserves careful reading. The only objection we can see in reprinting Beaumont's article by itself, for the use of ophthalmic surgeons, is that other articles in the book, notably those on pain and hysteria, require to be read with it. As a criticism, we would suggest that a little condensation would be advisable.

E. E. H.

\section{Ophtalmologie du Médecin Praticien. (Ophthalmology for the} General Practitioner.) Par Albert Terson. Paris VI ${ }^{\mathrm{e}}$ : Masson et Cie., 120 Boulevard St. Germain. 1916. Price 12 francs.

The object of this text-book of ophthalmology is to teach the general practitioner who has had no practical experience in an eye clinic, how to treat and to recognize some of the common diseases of the eye. The author points out that the family medical man cannot always escape giving an opinion upon a diseased eye, even if it be only to decide whether a specialist should be called in or not; if he has no knowledge of the subject, he will be liable to injure his reputation. We are inclined to think that, in this country at any rate, more reputations are lost by the possession of that dangerous "little knowledge" than by a confession of complete ignorance. The public to-day does not expect a general practitioner to be a specialist, and greatly prefers to be referred to an expert.

But if ' the general practitioner wish to acquire a working knowledge of eye diseases-and many succeed in their desire-there is only one way to act; he must work in an eye clinic regularly for a year or two. It is quite impossible to get a grasp of any clinical subject without personal contact with the patients.

What knowledge of diseases of the eye should a general practitioner possess? In the first place, he should learn sufficient of the use of the ophthalmoscope to enable him to employ it in his daily work, to assist in the diagnosis of general disease. A physician who cannot diagnose optic neuritis and albuminuric retinitis, is as badly handicapped as one who cannot hear a heart 
murmur. The ophthalmoscope is as essential as the stethoscope in the diagnosis of systemic disease. Secondly, the general practitioner ought to be able to recognize that an eye is so affected as to call for the attention of a specialist. Above all things, let him be instructed that vomiting and general malaise may be signs of acute glaucoma. All this should be taught to students, and no diploma should be issued to a man who has not this knowledge.

There are many details which could be criticized in the book, but it seems sufficient to point out the difficulty of teaching without practical experience.

We are at a loss to understand the objection the author has to the use of atropin in ulcers of the cornea. He cautions the reader against atropin, unless there be severe iritis. We are apt to regard this drug as the sheet-anchor of our treatment, and omit it only in the case of patients with a tendency to glaucoma.

No practical details are given for testing colour vision, and yet we should have thought that this was often the duty of a general practitioner who holds a railway appointment. Stilling's test and Holmgren's wools are mentioned, but not a word of caution is given as to the errors likely to be made with these tests. The colour perception lantern is not mentioned.

The use of three or four founts of type on each page is very teasing, and the illustrations are poor in comparison with those found in English and American books.

T. HARRISON BUTLER.

\section{The American Encyclopedia and Dictionary of Ophthalmology.}

Edited by CASEY A. WoOD, M.D., C.M., D.C.L., assisted by a large staff of collaborators. Volume X.- Lenicet to Muscles. Chicago : Cleveland Press. 1917.

Much as one would like to review in some detail the various important articles in this tenth volume of the "American Encyclopedia," which contains such subjects as lens and lenses (200 pages or thereby), life insurance and ophthalmology, light, localization of foreign bodies, magnet, ocular muscles, and others; yet at the present moment all these are overshadowed in importance by the article on military surgery of the eye by Lloyd Mills, of Los Angeles. The author has had the initial advantage of being at the time of his writing a neutral and has had access to German and Austrian literature; further, he was in the Austrian medical service. The result is an article in which through the combination of personal experience with literary talent we have an up-to-date summary of ocular, and to a large extent, of cerebral military surgery. It is probable that no other single article of equal comprehensiveness is to be found in the English language. 
The author commences by showing statistically the great increase in the proportion of killed to wounded in this war as compared with previous wars, and the similar increase of head and eye injuries. These unfortunate results are due not only to the increased power of armament, but also to the development of trench warfare. In the Chino-Japanese war of 1894 eye injuries formed 1.2 per cent. of all wounds, whereas in the present conflict it seems probable that Uhthoff's estimate of 8 per cent. will be nearly correct. The degree of prevention by metal helmets remains to be determined. Looking to the very large amount of blindness in previous wars from immediate enucleation or, alternatively, sympathetic ophthalmitis, the establishment of specialist centres and of skilled ophthalmologists " have proven of incalculable value in terms of vision and of life." The mere covering of bulbar wounds by a conjunctival flap has saved numerous eyes. A propos, there follows a section giving instruction in first aid (ocular) to military surgeons. One notes the omission of tabloid drugs from the equipment. From this the author passes on to the consideration of the conditions of employment of shrapnel, shells and bullets, the mechanism of their action, their characteristic effects upon the tissues of the head, and the general principles of the treatment of military wounds. In this last connection, and basing on "solidly established facts," Mills goes rather closely into the question of the use of antiseptic solutions which has been brought into such prominence by the researches of Sir Almroth Wright, Carrel, Dakin and others; precise directions for the making up of such solutions (Dakin's solution), are given. When ready for use Dakin's solution contains 0.5 per cent. of sodium hypochlorite with small amounts of neutral soda salts, and is practically isotonic with serum.

Wounds of the optic apparatus are no exceptions to the routine injection of prophylactic doses of antitetanic serum, usually 1,500 units subcutaneously. Gas bacillus infection of the orbital regions is rare. Most of the cases succumb in spite of free multiple incisions, the cause of death being meningeal complications.

A regional classification of the battle wounds of the optic apparatus follows. It is necessarily inexact, the author points out, because uncomplicated injuries of the eyes and optic apparatus are exceptional. A few points may be noticed.

Complete loss of the upper lid practically always means loss of the eye, even though this is uninjured, "although the use of a pedicled flap, backed with a conjunctival transplant from the eye of a recently killed donor and sutured temporarily to the lower lid, offers some slight chance of saving the eye; or, in many cases it will at least support a prothesis."

injuries of the lacrymal sac usually result in suppuration before they can be treated, and the sac must be excised, but if early 
treatment is possible, suture of the mucous membrane and skin in front of a hard rubber sound through the canaliculus and severed ends of the duct usually gives a patent duct. In injuries of the canaliculus Elschnig passes a fine hard rubber sound through the canaliculus from the punctum to the sac after silk sutures have been placed both on the conjunctival and skin surfaces of the injured lid. The sound is cut off an inch from the punctum and left in place for seven days with vaselined gauze between lid and sound.

Death from infection by the streptococcus, and rarely from B. perfringens or $\mathrm{B}$. tetanus, not infrequently follows crushing injuries of the bony orbit unless the dead, contused tissue is freely cut away, foreign bodies are removed and drainage is provided by gauze wicks wet with sodium hypochlorite solution. Such dressings must on no account be waterproofed, and must be frequently changed. All such cases receive antitetanic serum and a mixed vaccine with streptococcus predominating. Morestin details the successful treatment of a number of cases of traumatic defect of the upper orbital arches, as well as of frontal and other skull depressions or defects, by implanting or grafting the costal cartilages of the sixth, seventh and eighth ribs, trimmed to the required shape, the graft " taking" even when not autogenous.

It is pointed out that in bullet wounds through the orbit behind an intact anterior segment of the eye choroidal and retinal ruptures of types not usually seen in civil practice may exist. The typical concentric rupture is rare. The case is reported by Fleischer of a soldier who felt a blow on his head which caused his helmet to fall forward. The right eyeball lay in the hollow of the helmet, unruptured. A bullet had entered in front of the left ear and emerged in front of the right external canthus, leaving the bulbar conjunctiva as smoothly divided as though an ideal enucleation had been performed. Contrariwise, in bullet injury of the orbit the optic nerve is not always directly injured though the eye may be blind for the time being, due to concussion or contusion of the nerve, or to inflammatory reaction about the nerve.

Indirect injuries of the eyeball made up over 6 per cent. of all battle injuries of the eyes in present-day warfare. These are placed in two groups, namely (1) those which are purely the result of air contusion or air decompression, consequent upon the bursting of high explosive shells in the near neighbourhood, and (2) those in which the ocular coats have been injured by contusion, penetration or perforation of the orbital cone by projectile or other injury, with no possibility of contusion of the globe. Numerous examples are detailed. Retinal detachment forms a large proportion of such indirect injuries. In these cases there is usually no pain referred to the eye, which nearly always retains its normal external appearance. 
Discovery of the blindness, the other eye being sound, is generally accidental.

The author goes closely into the details of direct injuries of the eyes by bullets, by artillery projectiles and by the very numerous secondary missiles such as mud, dust, sand, gravel, fragments of rock, splinters of wood, metal and glass, and so on. There is a special paragraph on the subject of conjunctival flaps. "In all perforating wounds of the anterior segment of the eye the first indication is to close the wound and to protect the eye against infection by covering the wound with a conjunctival flap after as thorough a conjunctival toilet as possible." Cords says that "the living conjunctiva is the best antiseptic and the best antisepsis, and provided with such a bandage, the wounded soldier can be safely transported, reaching a specialist within a few days at the most and in time for enucleation should purulent inflammation have begun." Bahr recommends painting all the wounded tissues of the face, the lids and the anterior segment of the globe, with full strength tincture of iodine as the first treatment. Though this is done under thorough cocainization the resulting pain is severe and persistent, but infection is usually avoided so far as this has not taken place at the time of injury.

In dealing with traumatic cataract the author considers that in unskilled hands the only justifiable treatment is the immediate use of a conjunctival covering and dispatch of the patient to the specialist centre. He refers to the well-known view of Valude that soldiers suffering from traumatic cataract of one eye should not be operated on when the remaining eye is sound.

It is noteworthy that foreign bodies of the cornea or conjunctiva frequently produce the gravest consequences (Terrien), this being due to commotio oculi. Terrien believes that inflammatory, sensorial and nervous disorders occurring in these cases are part of a single "hystero-traumatic entity" or syndrome, in which the idea of simulation has no rightful existence. Foreign particles are not found in all these cases to explain the syndrome on the basis of direct mechanical trauma.

The subject of intra-ocular foreign bodies has been discussed so recently in this country-and indeed the opinions of British authors are here mentioned-that little reference to this need be made. It is interesting, nevertheless, to note some paragraphs which the author of this article has thought necessary to italicize. (1) "No intra-ocular search for a foreign body is justifiable without a careful Röntgenographic study of the eye and orbit from different angles." (2) "The presence of one or more small foreign bodies in the eye does not mean, of necessity, the loss of the eye or great reduction in its vision." (3) "The enormous experience of this war seems to show that the presence of any kind of a sterile foreign 
body in the eye has no relation to the production of sympathetic ophthalmitis. As a result of this war there are literally thousands of men going about with apparently innocuous particles of metal and of various forms of rock in one or both eyes, and with useful vision ranging up to $6 / 9$ or even better."

The rules for the prophylactic enucleation of wounded eyes seem to be very much the same as those referred to by British writers, and Lister's method of leaving a cuff of sclera is highly recommended.

In cases of unilateral traumatic exophthalmos which survive, in which the line of perforation is so clearly within the orbit as to exclude the possibility of arterio-venous aneurism in the cavernous sinus or other post-orbital lesions, the orbit should be incised and the cavity of the hematoma freed of clot and drained in order to anticipate the infection which so commonly follows localized orbital hæmorrhage.

A long section deals with lesions of the upper cranial nerves, and with those of the sympathetic in the neck, after which attention is focussed on concussion, blindness, and shell shock. The greater part of the literature quoted on the last-named subject is British, and further reference will not therefore be made here.

Among the ocular disorders largely or wholly evoked by physical and nervous exhaustion military night-blindness has been reported by a number of observers. Zade calls attention to the effect of actively military service in seriously reducing the visual acuity of men with uncorrected or imperfectly corrected anomalies of refraction, while Wietfeldt believes that the lack of vitamines in the food may be a factor in causing night-blindness.

The first part of the next section refers mainly to points in the treatment of cranial wounds as such and may be omitted here. The second part deals with the relations of projectile injuries of the skull and brain to the eyes. These relations "are established especially through increased intracranial pressure from many sources, through actual destruction of or injury to the visual cortical centres or the visual pathways and through local and general meningeal or encephalic infection. The ocular expressions of these injuries appear in the form of papilloedema and optic neuritis of all grades : muscular pareses, paralysis and conjugate deviations; and defect phenomena in the visual fields, ranging from total binocular blindness to tiny unilateral relative scotomata." This subject is then elaborated: one or two points only may be noticed. Progressive optic neuritis is a grave sign, the progress and not the mere fact of its existence being the important point. According to Adams, papillitis is not an indication for operation. The cases are not comparable with brain tumour. The change in the optic nerve simply means that something is wrong with the brain. 
To the quotation from Adams the writer of the article adds in italics, "If, therefore, no other symptom furnishes an indication for trephining, that of choked disc alone should not be an indication for opening the skull." The author produces evidence from various writers that it is a safe rule that all projectile wounds of the skull be kept under occasional observation for many months, as late infections are apt to occur.

Injuries of the frontal lobes are next dealt with, such war injuries forming no exception to the rule that greater liberties can be taken with this than with other parts of the brain. Eight cases of nystagmus recorded by Noehte after injury of the foot of the second frontal convolution are given in some detail and the conclusion reached that in these cases there was no uniform relation between the side of injury and the direction of ocular movement. Noehte believes that each centre consists of several parts, lesions of which lead to right or left nystagmus, or the combined involvement of which produces nystagmus in all directions. Certain ocular palsies resulting from wounds of the sinuses and especially of the superior longitudinal sinus, are then discussed.

Some dozen pages are required to deal with the important subject of injuries of the occipital region and the resulting ocular symptoms, central blindness "and all manner of permanent and transitory defects in the visual fields." This must be studied in the original : an abstract could do no justice to the cases recorded. One may draw special attention to the case of bilateral motor apraxia with disturbance of visual orientation reported by Smith and Holmes as following the passage of a twelve millimetre shrapnel bullet through the skull and brain, involving the supramarginal gyri.

From the section on ocular disorders and injuries of aviation one gains some slight idea of the frightful visual strain thrown on aviators. The following sections deal with "ocular diseases or recrudescences of old general, and ocular diseases " occurring in warfare, such as might be expected to follow similar strain or exposure in civil pursuits or avocations; and with malingering. In the former group epidemic nephritis finds a place, and the work of Foster Moore, Abercrombie and Jessop is referred to.

The final section deals with the relationship of visual acuity to military efficiency. The table, published in the Lancet, by Paterson and Traquair is reproduced and their work freely acknowledged. In discussing the disabling effect of Miners' nystagmus the British work by Ormond and by Harford is the source of information. Regarding the totally blind the author says that "the efforts of the Germans along this line are worthy of serious emulation in all countries, with due regard, of course, to the prevailing standards of living." The last few paragraphs deal with the training of those 
blinded in war. It is unfortunate that no account of St. Dunstan's Hostel has been included.

ERNEST THOMSON.

\section{CORRESPONDENCE}

\section{Atropin in Cataract Extraction}

\section{To the Editor of THE BRITISH JouRnAL OF Ophthalmology}

SIR,--In the course of reading the proofs of the December number of the BRITISH JOURNAL OF OPHTHALMOLOGY, I was interested in Mr. Fisher's advocacy of the use of atropin in cataract extraction. But surely, Sir, this is nothing new. It was done habitually by some of the best-known Continental operators, notably by Axenfeld, and by Hess, of Munich. I have seen both these surgeons operate with the atropin-dilated pupil. The following is a transcript of my notes taken at the time :-(1) "Extraction of cataract in a young adult by Axenfeld. Axenfeld has the pupil dilated either with homatropin an hour before, or with scopolamin two days before operation. (2) Hess. Extraction of senile cataract. The pupil has been dilated with atropin, one drop of 1 per cent. the night before and one drop on the morning of operation. The pupil is therefore fully dilated." The operative details were also given in my articles on Continental clinics, Ophthalmoscope, 1912. It is true that Fisher uses the atropin when the patient is on the table, and that more of the alkaloid may thus enter the eye, but the principle seems exactly the same as that employed in 1911 by the two surgeons named. Hess was at that time in Würzburg.

\section{Yours etc.,}

ERNEST THOMSON.

\section{To the Editor of The BRITISH Journal of Ophthalmology}

SIR,

I think Mr. Fisher's note on cataract extraction in this month's issue of your Journal should not pass unnoticed.

There appears to be little that is new under the sun, and it may interest $\mathrm{Mr}$. Fisher to hear that Lieut.-Col. Drake-Brockman told me at the International Ophthalmological Congress at Lucerne, some few years ago, that he systematically dilated the pupil before cataract extraction, that I myself do the same thing, and that Mr. Stanford Morton has for many years dropped a solution of atropin into the eye immediately after extraction : probably many others use it before or after in the same way. 